'Línea de Investigación

Salud y Calidad de Vida.

Escuela de Psicología,

Universidad Católica del Norte.

${ }^{2}$ Departamento de

Psicología, Universidad de Tarapacá, Arica, Chile.

aPsicólogo, Doctor en

Psicología Clínica y de la Salud.

bPsicóloga.

Este artículo es producto de un estudio financiado por la Comisión Nacional de Investigación Científica y Tecnológica del Gobierno de Chile - CONICYT, a través del proyecto FONDECYT 11100090, quienes no tuvieron influencia en el diseño del estudio, recolección, análisis o interpretación de los datos, ni en la preparación, revisión o aprobación del manuscrito.

Recibido el 29 de junio de 2012, aceptado el $13 \mathrm{de}$ junio de 2013

Correspondencia a: Dr. Alfonso Urzúa

Escuela de Psicología, Universidad Católica del Norte, Avenida Angamos 0610, Antofagasta, Chile. E-mail: alurzua@ucn.cl

\section{La valoración de la importancia en el autoreporte de la calidad de vida en la adultez}

\author{
ALFONSO URZÚA M. ${ }^{1, a}$, KRIST CORTÉS $^{1, b}$, CARRIE MAITA ${ }^{1, b}$, \\ KATHERINE OSORIO ${ }^{1, \mathrm{~b}}$, ALEJANDRA CAQUEO-URIZAR ${ }^{2, a}$
}

\section{Weight of specific domains on the overall quality of life according to the assessment of participants}

\begin{abstract}
Background: Usually quality of life self-reports are standard and consider that all the areas evaluated are equally important. Aim: To explore the influence of specific domains on the overall quality of life self-assessment. Material and Methods: The briefversion of the quality of life questionnaire developed by the World Health Organization (WHOQoL bref) was applied to 1229 participants aged 19 to 60 years (53\% women). The questionnaire explores four domains: physical and psychological health, social relationships and environment. At the end of each domain a new question asking the participant to assess the importance of the domain, using a scale of 1 (not important) to 5 (very important), was added. The analysis of data considered the assessment of the importance of each domain made by participants. The weight of each domain was analyzed using regression equations. Results: The physical health had the best evaluation followed by the social domain. The environmental domain received the lower evaluation. Among women, social domain was the best evaluated domain and among men, the physical health domain. According to participants, physical and psychological health had the higher weight. The environmental domain had the higher weight on the overall assessment of quality of life. Conclusions: According to participants, physical and psychological health are the most important aspects that influence overall quality of life.
\end{abstract}

(Rev Med Chile 2013; 141: 1010-1018).

Key words: Adult; Quality of life; Self-report.
L a Organización Mundial de la Salud definió la Calidad de Vida (CV) como la percepción individual de la posición en la vida en el contexto de la cultura y sistema de valores en el cual se vive y su relación con las metas, expectativas, estándares e intereses ${ }^{1}$. Operacionalmente, puede ser definida como el nivel percibido de bienestar derivado de la evaluación que realiza cada persona de elementos objetivos y subjetivos en distintas dimensiones de su vida ${ }^{2}$.

La investigación en esta área ha estado vinculada al desarrollo y evaluación de instrumentos y a estudiar los factores vinculados a la CV en áreas específicas, como el de la Calidad de Vida relacionada con la Salud (CVRS), definida como el nivel de bienestar derivado de la evaluación que la persona realiza de diversos dominios de su vida, considerando el impacto que éstos tienen en su estado de salud real o percibido ${ }^{3}$. Su estudio ha permitido, entre otras, dar lineamientos respecto al trabajo con enfermedades, en tanto decisiones clínicas o condiciones de vida ${ }^{4}$, optimizar el tratamiento de los pacientes además de conocer los requerimientos y la distribución de los recursos en una población determinada ${ }^{5}$. Los estudios de $\mathrm{CV}$ en población no enferma son más recientes $\mathrm{y}$ han estado centrados en pesquisar diversos factores que pueden incidir en la evaluación que 
la persona hace de su CV. Entre estos factores, se la ha vinculado a factores históricos, culturales y de clase social ${ }^{6}$, la edad ${ }^{7}$, una vida social activa ${ }^{8}$, el desempleo o una imagen corporal pobre ${ }^{9}$, la presencia de morbilidad psicológica ${ }^{10}$, cambios sociales ${ }^{11}$ o el nivel de educación, estado civil y la confianza en la seguridad social ${ }^{12}$.

En Chile, los estudios enfatizan la evaluación de la CVRS ${ }^{13-17}$, en sitios geográficos específicos y las diferencias de medidas con otros grupos ${ }^{18,19}$.

Pese al avance en la investigación, los estudios se han centrado en describir o relacionar la CV con factores que pueden influir en un mejor o peor autoreporte, y no en comprender de qué manera las personas realizan esta evaluación, esto es, los procesos socio cognitivos que pudiesen estar incidiendo en esta ${ }^{2}$. Uno de estos procesos es la importancia que las personas le atribuyen a cada uno de los dominios que inciden en la percepción de la CV. Dado que cada persona asigna distinto valor a cada ámbito de su vida cotidiana ${ }^{20}$, es esperable encontrar diferencias al evaluar la CV, cuando se pondera la evaluación de la persona por la importancia que esta le asigna de manera individual, toda vez que evaluamos con modelos que incluyen dominios estandarizados, en donde partimos del supuesto que todas las áreas son igual de importantes para las personas.

En este marco, esta investigación tuvo por objetivo analizar las diferencias existentes en la evaluación de la CV y de sus distintos dominios, al incorporar la valoración de la importancia del dominio.

\section{Materiales y Método}

Esta investigación se realizó bajo un diseño de tipo no experimental, analítico transversal.

\section{Participantes}

A través de un muestreo no probabilístico por conveniencia, de tipo estratificado con similar distribución por sexo y edad, se encuestaron 1.229 adultos, funcionarios o concurrentes de organizaciones públicas y sociales de la ciudad de Antofagasta, Chile, tales como universidades, empresas privadas, instituciones gubernamentales, salas de espera de INP, FONASA, bancos, etc. De los participantes el 50,3\% eran mujeres. Las edades se clasificaron en rangos: 19 a $24(32,5 \%)$,
Tabla 1. Distribución de participantes por sexo y rango de edad

\begin{tabular}{|lcccc|}
\hline & $\begin{array}{c}\text { 19 a } \mathbf{2 4} \\
\text { años }\end{array}$ & $\begin{array}{c}\mathbf{2 5} \text { a } \mathbf{3 9} \\
\text { años }\end{array}$ & $\begin{array}{c}\mathbf{4 0} \text { a } \mathbf{6 0} \\
\text { años }\end{array}$ & Total \\
\hline Hombre & 200 & 200 & 211 & 611 \\
Mujer & 200 & 186 & 232 & 618 \\
\hline Total & 400 & 386 & 443 & 1.229 \\
\hline
\end{tabular}

25 a 39 años (31,4\%) y 40 a 60 años (36\%) (Tabla 1). Del total de personas, 1.064 declararon vivir con una enfermedad crónica $(86,6 \%)$. De estas, las más frecuentes fueron la hipertensión arterial $(3,0 \%)$, diabetes mellitus $(2,3 \%)$ y asma $(1,6 \%)$. No existió corroboración clínica de la enfermedad crónica, se consideró sólo el autoreporte de enfermedad.

\section{Instrumento}

Para evaluar la CV se utilizó el WHOQOLBREF, compuesto por 26 preguntas. Una pregunta inquiere sobre la evaluación global de la $\mathrm{CV}$, una sobre la satisfacción con la salud y las 24 restantes son agrupadas en cuatro dominios: Salud física, Salud psicológica, Relaciones sociales y Ambiente ${ }^{21}$. Las respuestas son puntuadas de 1 a 5 y sumadas por dominio, en donde a mayor puntuación mejor CV. La escala WHOQOL-BREF ha sido validada en diversos estudios alrededor del mundo ${ }^{22}$, incluyendo su versión en castellano ${ }^{23}$.

\section{Procedimientos}

Una vez obtenida la aprobación del Comité de Ética de CONICYT, los participantes completaron los cuestionarios, firmando previamente un consentimiento informado. Los datos fueron ingresados a una planilla construida en SPSS 17.0.

A fin de valorar el grado de importancia que le asignaban los participantes a cada uno de los ámbitos evaluados, se agregó a cada ítem que compone la WHOQOL-BREF una pregunta en donde cada participante valoró de 1 a 5 el grado de importancia que para él tenía el ámbito sobre el cual se estaba inquiriendo (opciones desde 1 No es importante a 5 Muy importante). A fin de mejorar la interpretación de los datos, se transformaron los puntajes obtenidos con base a los valores ponderados a una escala de 0 a 100.

Se analizó de manera descriptiva la CV y sus 
dimensiones, segmentando por sexo y por rango de edad, luego se aplicó t de Student para verificar las diferencias significativas según sexo y ANOVA para los rangos de edad. Finalmente, se realizaron regresiones a fin de conocer el peso de los dominios en la evaluación de la CV. Se consideró como VD los resultados de la evaluación global que la persona realiza sobre su CV (pregunta 1). Como variables independientes (VI) se consideraron los dominios evaluados y que son construidos con base a los reactivos restantes. Este último análisis se hizo estratificando la muestra por sexo, edad y presencia/ausencia de enfermedad crónica (EC). Los mismos procedimientos se aplicaron para analizar la CV ponderada por importancia.

\section{Resultados}

\section{Calidad de vida y sexo}

En la Tabla 2 se muestran los resultados totales de CV y de cada dominio, para el total de la muestra y por sexo considerando los puntajes brutos. A nivel de la muestra total, el dominio mejor evaluado correspondió al físico, seguido por el social y el peor evaluado al ambiental. Esto difiere al separar por sexo, ya que en las mujeres el mejor evaluado fue el dominio social, manteniéndose el ambiental como el peor evaluado. Los hombres tuvieron medias significativamente mayores que las mujeres en los dominios físico $\left(t_{(1225)}=6,752\right.$; $\mathrm{p}<, 01)$, psicológico $\left(t_{(1226)}=7,257 ; \mathrm{p}<, 01\right) \mathrm{y}$ ambiental $\left(t_{(1223)}=3,454 ; \mathrm{p}<, 05\right)$.

Al considerar los puntajes ponderados por importancia (Tabla 3), los dominios mejor evaluados en el caso de los hombres fueron el físico y el psicológico, en tanto en las mujeres lo fue el físico. La media del dominio psicológico en los hombres fue la única significativamente mayor que la de las mujeres $\left(t_{(1222)}=3,101 ; \mathrm{p}<, 05\right)$.

Tal como se puede observar en la Tabla 4, al considerar la evaluación global de la $\mathrm{CV}$, la varianza explicada por los dominios varió entre hombres y mujeres y aumentó al ponderar por la importancia, alcanzando hasta $35 \%$ en el caso de la CV ponderada por la importancia en el grupo de hombres.

En la Tabla 5 se muestran las variables significativas en cada uno de los modelos estudiados. Tal como se aprecia, las VI significativas en todos los modelos fueron los dominios psicológico y ambiental, cambiando el peso que tiene cada una de estas en la CV al valorar o no por importancia.

Tabla 2. Puntaje calidad de vida para muestra total y segmentado por sexo

\begin{tabular}{|c|c|c|c|c|c|c|c|c|c|}
\hline & \multicolumn{3}{|c|}{ Total } & \multicolumn{3}{|c|}{ Hombres } & \multicolumn{3}{|c|}{ Mujeres } \\
\hline & $\mathbf{n}$ & $\mathbf{M}$ & DE & n & $\mathbf{M}$ & DE & $\mathbf{n}$ & $\mathbf{M}$ & DE \\
\hline CV global & 1.229 & 3,50 & 0,759 & 611 & 3,63 & 0,77 & 618 & 3,46 & 0,76 \\
\hline Físico & 1.227 & 65,47 & 13,76 & 610 & 68,1 & 12,97 & 617 & 62,88 & 14,03 \\
\hline Psicológico & 1.228 & 63,99 & 14,14 & 611 & 66,87 & 13,85 & 617 & 61,14 & 13,84 \\
\hline Social & 1.229 & 65,06 & 16,96 & 611 & 65,63 & 16,92 & 618 & 64,51 & 17,00 \\
\hline Ambiental & 1.225 & 57,75 & 12,39 & 610 & 58,67 & 11,87 & 615 & 56,53 & 12,78 \\
\hline
\end{tabular}

Tabla 3. Medias CV y dominios ponderados por importancia en muestra total y por sexo

\begin{tabular}{|c|c|c|c|c|c|c|c|c|c|}
\hline & \multicolumn{3}{|c|}{ Total } & \multicolumn{3}{|c|}{ Hombres } & \multicolumn{3}{|c|}{ Mujeres } \\
\hline & n & $\mathbf{M}$ & DE & n & M & DE & n & $\mathbf{M}$ & DE \\
\hline CV global & 1.228 & 15,89 & 4,505 & 610 & 15,89 & 4,62 & 618 & 15,91 & 4,38 \\
\hline Físico & 1.222 & 64,92 & 12,25 & 609 & 65,53 & 12,17 & 613 & 64,31 & 12,32 \\
\hline Psicológico & 1.224 & 64,39 & 12,68 & 609 & 65,52 & 13,12 & 615 & 63,28 & 12,14 \\
\hline Social & 1.228 & 63,02 & 16,26 & 610 & 63,27 & 16,27 & 618 & 62,78 & 16,31 \\
\hline Ambiental & 1.220 & 56,17 & 10,67 & 607 & 56,39 & 10,72 & 613 & 55,96 & 10,62 \\
\hline
\end{tabular}


La valoración de la importancia en la calidad de vida - A. Urzúa et al

Tabla 4. Varianza explicada por las variables independientes para cada modelo

\begin{tabular}{|lcccc|}
\hline Modelo & $\mathbf{R}$ & $\mathbf{R}^{\mathbf{2}}$ & $\mathbf{R}^{\mathbf{2}}$ ajustada & Error estándar estimado \\
\hline CV Hombres &, 550 &, 302 &, 297 &, 652 \\
CV por importancia hombres &, 596 &, 355 &, 351 & 3,74 \\
CV Mujeres &, 533 &, 285 &, 280 &, 625 \\
CV por importancia mujeres &, 548 &, 301 &, 296 & 3,67 \\
\hline
\end{tabular}

Tabla 5. Variables significativas a modelos de CV en hombres y mujeres ponderados y no por importancia

\begin{tabular}{|c|c|c|c|c|c|c|}
\hline & & \multicolumn{2}{|c|}{ CNE } & CE & \multirow[b]{2}{*}{$\mathbf{t}$} & \multirow[b]{2}{*}{$\mathbf{p}$} \\
\hline & & B & EE & Beta & & \\
\hline \multirow[t]{4}{*}{ CV Hombres } & (Constante) & 1,132 & 163 & & 6,947 &, 000 \\
\hline & Dominio psicológico &, 012 & ,003 & 211 & 4,354 &, 000 \\
\hline & Dominio social &, 004 &, 002 & ,089 & 2,009 &, 045 \\
\hline & Dominio ambiental & 023 &, 003 & ,345 & 8,202 & 000 \\
\hline \multirow[t]{3}{*}{ CV Mujeres } & (Constante) & 1,400 & 138 & & 10,129 &, 000 \\
\hline & Dominio psicológico & ,014 &, 002 & ,256 & 5,439 &, 000 \\
\hline & Dominio ambiental &, 016 & ,002 & ,277 & 6,424 & 000 \\
\hline \multirow{3}{*}{$\begin{array}{l}\text { CV por importancia } \\
\text { hombres }\end{array}$} & (Constante) &,- 218 & ,933 & &,- 233 & 816 \\
\hline & Dominio psicológico & ,097 & 018 & ,275 & 5,327 &, 000 \\
\hline & Dominio ambiental & 144 &, 019 & ,333 & 7,452 &, 000 \\
\hline \multirow{3}{*}{$\begin{array}{l}\text { CV por importancia } \\
\text { mujeres }\end{array}$} & (Constante) & 1,578 & ,915 & & 1,725 & 085 \\
\hline & Dominio psicológico & ,100 & 018 & 277 & 5,452 &, 000 \\
\hline & Dominio ambiental &, 096 & 019 & 233 & 5,024 & ,000 \\
\hline
\end{tabular}

$\mathrm{CE}=$ Coeficiente estandarizado; $\mathrm{CNE}=$ Coeficiente no estandarizado; $\mathrm{EE}=$ Error estándar.

Tabla 6. Puntajes para calidad de vida en muestra segmentada en rangos de edad

\begin{tabular}{|lccccccccc|}
\hline & \multicolumn{3}{c}{$\mathbf{1 9} \mathbf{~ a ~ 2 4}$ años } & \multicolumn{3}{c}{$\mathbf{2 5}$ a $\mathbf{3 9}$ años } & \multicolumn{3}{c|}{$\mathbf{4 0}$ a $\mathbf{6 0}$ años } \\
& $\mathbf{n}$ & $\mathbf{M}$ & $\mathbf{D E}$ & $\mathbf{n}$ & $\mathbf{M}$ & $\mathbf{D E}$ & $\mathbf{n}$ & $\mathbf{M}$ & $\mathbf{D E}$ \\
CV Global & 400 & 3,52 & 0,77 & 386 & 3,54 & 0,72 & 443 & 3,44 & 0,77 \\
Dominio físico & 399 & 66,27 & 13,12 & 386 & 67,16 & 13,26 & 442 & 62,29 & 14,48 \\
\hline Dominio psicológico & 400 & 63,72 & 14,99 & 386 & 65,85 & 12,86 & 442 & 62,62 & 14,26 \\
\hline Dominio social & 400 & 67,01 & 17,09 & 386 & 66,86 & 16,32 & 443 & 61,74 & 16,92 \\
\hline Dominio ambiental & 398 & 58,00 & 12,7 & 385 & 58,22 & 12,32 & 442 & 57,12 & 12,17 \\
\hline
\end{tabular}

\section{Calidad de vida y edad}

Considerando los valores brutos por edad (Tabla 6), se encontraron diferencias estadísticamente significativas en el dominio físico $\left(F_{(2,1224)}=9,222 ; \mathrm{p}\right.$ $<, 01)$, donde la media de los rangos de edad de 19 a 24 y de 25 a 39 fueron significativamente mayores que la del rango de 40 a 60 años $(\mathrm{p}<, 01$ en ambas), en el psicológico $\left(F_{(2,1225)}=5,537 ; \mathrm{p}<, 05\right)$, en donde la media del rango entre 25 a 39 años fueron significativamente mayor que el de 40 a 60 años $(\mathrm{p}<, 05)$ y en el dominio social $\left(F_{(2,1226)}=13,554\right.$; $\mathrm{p}<, 01)$, en donde la media de los rangos entre 19 a 24 y entre 25 a 39 fueron significativamente mayores que la media del rango entre 40 a 60 años ( $\mathrm{p}<, 01$ en ambas diferencias).

Al analizar los datos ponderados por im- 
portancia (Tabla 7), se encontraron diferencias estadísticamente significativas en los dominios físico $\left(F_{(2,1219)}=8,173 ; \mathrm{p}<, 01\right)$, en donde la media del rango de edad entre 25 a 39 años fue mayor que el de 40 a 60 años (p <,01); psicológico $\left(F_{(2,1221)}=6,578 ; \mathrm{p}<, 05\right)$, en donde la media del rango entre 25 a 39 fue significativamente mayor que la de los otros dos grupos de edad ( $\mathrm{p}<, 05$ en ambas comparaciones); social $\left(F_{(2,1225)}=15,087\right.$; $\mathrm{p}<, 01)$, en donde la media del rango entre 40 a 60 años fue significativamente menor que la de los otros dos ( $\mathrm{p}<, 01$ en ambas comparaciones) y ambiental $\left(F_{(2,1217)}=3,623 ; \mathrm{p}<, 05\right)$, en donde la media del rango de 25 a 39 años fue significativamente mayor que la del grupo de edad de 40 a 60 años $(\mathrm{p}<, 05)$.

La Tabla 8 muestra que la varianza de la CV explicada por las dimensiones varía en los distintos rangos de edad, alcanzando hasta $36 \%$ en el caso de la CV ponderada por la importancia en el grupo de 40 a 60 años.

La Tabla 9 muestra las variables significativas en cada uno de los modelos estudiados. Se encontró que las VI significativas en todos los modelos fueron los dominios psicológico y ambiental, con excepción del rango de edad entre 19 a 24 años, en donde también fue significativa al modelo la variable dominio social. Cabe mencionar que el valor más alto en el aporte de las variables a los modelos, lo constituyó el dominio ambiental en la mayoría de los rangos.

\section{Calidad de vida y enfermedad crónica (EC)}

En la Tabla 10 se muestran los valores obtenidos en la evaluación global de la CV y los diversos dominios. Considerando los puntajes brutos, se observa que entre los que reportan una EC, el dominio mejor evaluado fue el social, en tanto en los que no reportan una $\mathrm{EC}$ lo fue el físico.

Se encontraron diferencias estadísticamente significativas en los dominios físico $\left(t_{(1225)}=9,066\right.$; $\mathrm{p}<, 01)$, psicológico $\left(t_{(1226)}=5,083 ; \mathrm{p}<, 01\right)$, social $\left(t_{(1227)}=4,459 ; \mathrm{p}<, 01\right)$ y ambiental $\left(t_{(1223)}=2,929\right.$; $\mathrm{p}<, 05)$. La diferencia en la evaluación global de la CV también fue significativa $\left(t_{(1227)}=5,013\right.$; $\mathrm{p}<, 01)$.

$\mathrm{Al}$ analizar los valores ponderados por importancia, el dominio mejor evaluado entre los que reportan una EC fue el psicológico, en tanto en los que no, el físico.

Se encontraron diferencias estadísticamente significativas en los dominios físico $\left(t_{(1220)}=6,327\right.$; $\mathrm{p}<, 01)$, psicológico $\left(t_{(1222)}=3,905 ; \mathrm{p}<, 01\right)$, social $\left(t_{(1226)=} 4,626 ; \mathrm{p}<, 01\right) \mathrm{y}$ ambiental $\left(t_{(1218)}=2,224\right.$;

Tabla 7. Medias CV y dominios ponderados por importancia por rango de edad

\begin{tabular}{|c|c|c|c|c|c|c|c|c|c|}
\hline & \multicolumn{3}{|c|}{19 a 24 años } & \multicolumn{3}{|c|}{25 a 39 años } & \multicolumn{3}{|c|}{40 a 60 años } \\
\hline & $\mathbf{n}$ & $\mathbf{M}$ & DE & $\mathbf{n}$ & $\mathbf{M}$ & DE & $\mathbf{n}$ & M & DE \\
\hline CV Global & 400 & 15,84 & 4,57 & 385 & 16,18 & 4,32 & 443 & 15,702 & 4,59 \\
\hline Dominio físico & 399 & 64,8 & 11,91 & 381 & 66,83 & 11,71 & 442 & 63,39 & 12,81 \\
\hline Dominio psicológico & 398 & 63,98 & 12,99 & 385 & 66,25 & 11,85 & 441 & 63,14 & 12,94 \\
\hline Dominio social & 399 & 64,77 & 15,95 & 386 & 65,06 & 15,81 & 443 & 59,67 & 16,41 \\
\hline Dominio ambiental & 397 & 55,99 & 10,61 & 384 & 57,32 & 10,55 & 439 & 55,34 & 10,76 \\
\hline
\end{tabular}

Tabla 8. Varianza explicada por las variables independientes para cada modelo

\begin{tabular}{|lcccc|}
\hline Modelo & $\mathbf{R}$ & $\mathbf{R}^{\mathbf{2}}$ & $\mathbf{R}^{\mathbf{2}}$ ajustada & Error estándar estimado \\
CV 19-24 años &, 524 &, 274 &, 267 &, 660 \\
\hline CV por importancia 19-24 años &, 543 &, 295 &, 288 & 3,84 \\
CV 25-39 años &, 561 &, 315 &, 308 &, 600 \\
CV por importancia 25-39 años &, 566 &, 321 &, 314 & 3,58 \\
CV 40-60 años &, 546 &, 298 &, 291 &, 653 \\
CV por importancia 40-60 años &, 604 &, 365 &, 359 & 3,69 \\
\hline
\end{tabular}


La valoración de la importancia en la calidad de vida - A. Urzúa et al

$\mathrm{p}<, 05)$. La diferencia en la evaluación global de la CV también fue significativa $\left(t_{(1226)}=3,253\right.$; $\mathrm{p}<, 05)$.

Tanto al ponderar como no por importancia, las medias de los que no reportan una EC son superiores a las que lo sí lo hacen.
La Tabla 11 muestra la varianza explicada por los diversos dominios, la cual varía según la presencia o no de EC, alcanzando hasta $34 \%$ en el caso de la CV en los reportan EC, independiente o no del tipo de puntaje utilizado.

La Tabla 12 muestra que la VI significativa

Tabla 9. Variables significativas a modelos de CV por rangos de edad

\begin{tabular}{|c|c|c|c|c|c|c|}
\hline & & & VE & CE & & \\
\hline & & B & Std. Error & Beta & $\mathbf{t}$ & Sig. \\
\hline CV 19 a 24 años & (Constante) & 1,413 & 194 & & 7,297 &, 000 \\
\hline & Dominio psicológico & 010 &, 003 & , 189 & 3,227 & 001 \\
\hline & Dominio social &, 007 &, 002 & , 155 & 2,907 &, 004 \\
\hline & Dominio ambiental & ,019 &, 003 & 314 & 6,019 &, 000 \\
\hline CV 19 a 24 años por & (Constante) & 1,028 & 1,199 & &, 857 & ,392 \\
\hline importancia & Dominio psicológico &, 074 &, 022 & ,211 & 3,401 & 001 \\
\hline & Dominio ambiental &, 114 &, 024 &, 267 & 4,702 &, 000 \\
\hline CV 25 a 39 años & (Constante) & 1,312 & ,189 & & 6,948 &, 000 \\
\hline & Dominio psicológico & 013 &, 003 & ,230 & 4,060 &, 000 \\
\hline & Dominio ambiental &, 023 &, 003 & ,398 & 7,194 & 000 \\
\hline CV por importancia & (Constante) &, 850 & 1,220 & & 697 & 486 \\
\hline 25 a 39 años & Dominio psicológico &, 090 &, 023 & ,246 & 3,929 &, 000 \\
\hline & Dominio ambiental & , 146 &, 024 & ,356 & 5,995 &, 000 \\
\hline CV 40 a 60 años & (Constante) & 1,234 &, 168 & & 7,324 & 000 \\
\hline & Dominio psicológico &, 015 &, 003 & ,269 & 4,306 & 000 \\
\hline & Dominio ambiental &, 016 &, 003 &, 252 & 4,956 &, 000 \\
\hline CV por importancia & (Constante) & ,273 & 1,031 & &, 265 & 791 \\
\hline 40 a 60 años & Dominio psicológico &, 128 &, 023 & ,359 & 5,661 &, 000 \\
\hline & Dominio ambiental &, 106 &, 022 & ,249 & 4,779 &, 000 \\
\hline
\end{tabular}

$\mathrm{CE}=$ Coeficiente estandarizado; $\mathrm{CNE}=$ Coeficiente no estandarizado.

Tabla 10. Dominios evaluados según presencia de enfermedad crónica

\begin{tabular}{|c|c|c|c|c|c|c|c|c|c|c|c|c|}
\hline & \multicolumn{6}{|c|}{ Valores brutos } & \multicolumn{6}{|c|}{ Valores ponderados por importancia } \\
\hline & \multicolumn{3}{|c|}{ Reporta EC } & \multicolumn{3}{|c|}{ No reporta EC } & \multicolumn{3}{|c|}{ Reporta EC } & \multicolumn{3}{|c|}{ No reporta EC } \\
\hline & $\mathbf{n}$ & $\mathbf{M}$ & DE & $\mathbf{n}$ & M & DE & $\mathbf{n}$ & $\mathbf{M}$ & DE & $\mathbf{n}$ & $\mathbf{M}$ & DE \\
\hline CV global & 165 & 3,22 & .752 & 1.064 & 3,54 &, 752 & 165 & 14,84 & 4,49 & 1.063 & 16,06 & 4,49 \\
\hline Físico & 165 & 56,73 & 14,37 & 1.062 & 66,84 & 13,16 & 164 & 59,38 & 12,26 & 1.058 & 65,78 & 12,03 \\
\hline Psicológico & 164 & 58,82 & 14,49 & 1.064 & 64,79 & 13,92 & 164 & 60,82 & 12,94 & 1.060 & 64,95 & 12,56 \\
\hline Social & 165 & 59,63 & 17,52 & 1.064 & 65,91 & 16,73 & 165 & 57,62 & 16,82 & 1.063 & 63,87 & 16,01 \\
\hline Ambiental & 164 & 55,12 & 13,31 & 1.061 & 58,16 & 12,20 & 164 & 54,46 & 11,23 & 1.056 & 56,45 & 10,56 \\
\hline
\end{tabular}

Tabla 11. Varianza explicada por las variables independientes para cada modelo

\begin{tabular}{|lcccc|}
\hline Modelo & $\mathbf{R}$ & $\mathbf{R}^{\mathbf{2}}$ & $\mathbf{R}^{\mathbf{2}}$ ajustada & Error estándar estimado \\
CV Crónicos &, 599 &, 359 &, 342 &, 611 \\
CV por importancia crónicos &, 603 &, 363 &, 347 & 3,64 \\
CV No Crónicos &, 519 &, 270 &, 267 &, 642 \\
CV por importancia no crónicos &, 562 &, 315 &, 313 & 3,72 \\
\hline
\end{tabular}


Tabla 12. Variables significativas a modelos de CV por presencia/ausencia de EC

\begin{tabular}{|c|c|c|c|c|c|c|}
\hline & & & NE & CE & & \\
\hline & & B & Std. Error & Beta & $\mathbf{t}$ & Sig. \\
\hline CV No crónicos & (Constante) & 1,402 &, 119 & & 11,826 &, 000 \\
\hline & Dominio psicológico &, 013 &, 002 &, 235 & 6,501 &, 000 \\
\hline & Dominio ambiental & 019 &, 002 &, 314 & 9,687 &, 000 \\
\hline CV por importancia no crónicos & (Constante) & 875 & ,723 & & 1,210 &, 227 \\
\hline & Dominio psicológico & ,098 & 014 & ,273 & 7,113 &, 000 \\
\hline & Dominio ambiental &, 122 &, 015 &, 287 & 8,334 &, 000 \\
\hline CV Crónicos & (Constante) & 1,129 & ,229 & & 4,929 & 000 \\
\hline & Dominio ambiental &, 020 &, 005 & ,352 & 4,011 &, 000 \\
\hline CV por importancia crónicos & (Constante) & ,692 & 1,581 & &, 438 & ,662 \\
\hline & Dominio psicológico & ,090 &, 037 & ,258 & 2,461 &, 015 \\
\hline & Dominio ambiental & 121 & ,037 & ,303 & 3,264 &, 001 \\
\hline
\end{tabular}

$\mathrm{CE}=$ Coeficiente estandarizado; $\mathrm{CNE}=$ Coeficiente no estandarizado.

en todos los modelos fue el dominio ambiental, seguido por el psicológico, significativo en tres de los cuatro modelos.

\section{Discusión}

Los resultados evidencian que si bien no existe diferencia en la evaluación que hacen hombres y mujeres de su CV general, como ha sido reportado en estudios anteriores ${ }^{24-26}$, existen diferencias en la evaluación por dominios. Los hombres evalúan mejor el dominio físico a diferencia de las mujeres que evalúan mejor el dominio social. En los hombres probablemente la evaluación esta ligada a que éstos sólo perciben malestar físico si éste llega a ser grave ${ }^{27}$, en tanto las mujeres evalúan mejor el dominio social debido a la construcción de género que les permite tener mayor libertad y posibilidades en cuanto a sociabilización se refiere.

Al incorporar la importancia, ambos sexos evalúan mejor el dominio físico. Esta diferencia en los resultados podría estar ligada a la importancia que las mujeres dan a la imagen corporal y al poder que puede tener la imagen física o también a la conexión y preocupación que las mujeres tienen con su cuerpo.

El dominio con mayor peso al evaluar la CV fue el ambiental, en ambos sexos y ponderado o no por importancia. Esto podría estar relacionado a la valoración de elementos externos a la persona, además de las condiciones locales que estarían determinando las condiciones de vida de los participantes, ya que en la ciudad de Antofagasta se encuentran, por ejemplo, altos índices de contaminación, relacionados a la industrialización de la ciudad, además del ritmo de vida propio de la urbanización. Otro de los dominios con mayor peso en la CV fue el dominio psicológico, evidenciando la importancia que las personas otorgan al sentirse bien consigo mismas y a la ausencia de estados emocionales negativos. El estado emocional ha sido reportado en otras investigaciones como uno de los factores que más relevancia adquieren al momento de evaluar una persona su CV global ${ }^{28}$.

Cuando evaluamos CV por rangos de edad, la evaluación cambia al ser ponderada por importancia. El dominio mejor evaluado en el rango de 19 a 24 años es el social, posiblemente dado que los participantes en su mayoría fueron estudiantes universitarios que viven procesos de socialización intensos, debido a la alta demanda de redes sociales propias del contexto universitario. El rango de 25 a 39 años tiene como dominio mejor evaluado el físico, probablemente porque a esta edad (donde no se es ni joven, ni adulto mayor) existe un mayor compromiso con el cuerpo y una mayor conciencia de éste, por tanto la evaluación referida al mismo mejora. Posiblemente exista mayor conocimiento de lo físico, lo que daría como resultado mayor satisfacción a lo que éste se refiere.

Al ponderar por importancia, en los tres rangos de edad el dominio mejor evaluado es el físico, quizás porque este dominio se trasforma en la realidad más inmediata a la persona, con la cual 
interactúa con los otros dominios. Probablemente, las actuales condiciones sociales, y el uso de las nuevas tecnologías acentúen la valoración de este dominio, en cuanto las personas ven más expuesta su imagen.

Las medias del rango de 40 a 60 años son siempre significativamente menores en los diferentes dominios en comparación son los otros dos rangos, posiblemente influenciadas por el deterioro tanto a nivel biológico como cognitivo propio de la entrada a la adultez mayor, sumado a esto que el estado físico pudiese influir en un menor desempeño a nivel laboral, lo cual podría influir en las relaciones de pareja y familiares.

Entre las personas que no reportan una EC el dominio mejor evaluado es el físico, en tanto las personas que si reportaron una EC es el social, posiblemente por el apoyo que encuentran en este dominio, en especial en el área familiar que facilitan el ajuste a los cambios producidos por la EC. Todas las medias son menores en las personas con EC, lo que reafirma la hipótesis de que esta influye en todas dimensiones de la CV de una persona ${ }^{29}$.

Al ponderar por importancia, el dominio mejor evaluado entre las personas con EC ya no es el físico, sino el psicológico, posiblemente por su vinculación al control que la persona pueda sentir sobre su enfermedad, o al sentimiento de efectividad del tratamiento sugerido para ésta ${ }^{26}$. El dominio ambiental se repite como la variable más significativa, posiblemente ligada al acceso a los servicios de salud, y a facilidades o dificultades relativas a éstos.

No se han encontrado estudios similares que permitan analizar estos resultados a la luz de otros hallazgos, abriéndose nuevas líneas de investigación. Se sugiere que en futuros estudios se amplié la muestra con el fin de estratificar variables, para conocer las diferencias existentes entre sexos en relación a rangos de edad, respondiendo así a las siguientes preguntas: ¿Existe diferencia entre hombres y mujeres a medida que avanza la edad? En cuanto a las personas con EC, se propone aplicar la encuesta también al cuidador o familiar cercano, para así comparar calidad de vida bruta y ponderada por dominio cuando la condición de enfermo es evaluado por un externo, suprimiendo los grados de deseabilidad social al comparar ambas encuestas, además de conocer el pronóstico y el proceso de aceptación de enfermedad que lleva la persona con EC.

\section{Referencias}

1. The WHOQOL Group. The World Health Organization Quality of Life Assessment (WHOQOL): position paper from the World Health Organization. Soc Sci Med 1995; 41: 1403-09.

2. Urzúa A, Caqueo-Urízar A. Calidad de vida: una revisión teórica del concepto. Terapia Psicológica 2012; 30: 61-71.

3. Urzúa A. Calidad de vida relacionada con la salud: Elementos conceptuales. Rev Med Chile 2010; 138: 358-65.

4. Montazeri A. Health-related quality of life in breast cancer patients: a bibliographic review of the literature from 1974 to 2007. Journal of Experimental \& Clinical Cancer Research 2008; 27: 1-32.

5. Ivanovic F. Calidad de vida en la epilepsia. Revista Chilena de Epilepsia 2005; 4: 1-7.

6. De Souza M, De Araujo Z, Marchiori P. Qualidade de vida e saúde: um debate necessário. Ciência \& Saúde Colectiva 2000; 5: 7-18.

7. Urzúa A, Bravo M, Ogalde M, Vargas C. Factores vinculados a la calidad de vida en la adultez mayor. Rev Med Chile 2011; 139: 1006-14.

8. Oliveira K, Moreno M, Oliveira Lopes D, Pinto C. Factors associated with health-related quality of life for adults with stroke sequelae. Arq Neuropsiquiatr 2011; 69: 371-76.

9. Taira N, Shimozuma K, Shiroiwa T, Ohsumi S, Kuroi $\mathrm{K}$, Saji S, et al. Associations among baseline variables, treatment-related factors and health-related quality of life 2 years after breast cancer surgery. Breast Cancer Res Treat 2011; 128: 735-47.

10. Briongo L, Bachiller P, Palacios T, González M, Eiros J. Assessment of factors influencing health-related quality of life in HIV-infected patients. HIV Medicine 2011; 12: 22-30.

11. Chau-kui C, Kwok L. Ways that social change predicts personal Quality of Life. Soc Indic Res 2010; 96: 459-77.

12. Oztürk A, Tarsuslu T, Sertel E, Yümin M. The relationship between physical, functional capacity and quality of life (QoL) among elderly people with a chronic disease. Archives of Gerontology and Geriatrics 2011; 53: 278-83.

13. Herrera A, Arriagada J, González C. Calidad de vida y función sexual en mujeres postmenopáusicas con incontinencia urinaria. Actas urológicas españolas 2008; 32: 624-28.

14. Aguirre A, Benvenuto G, Urrutia M. Calidad de vida en mujeres con síndrome de ovario poliquístico. Rev Chil Obstet Ginecol 2005; 70 (2): 103-07.

15. Caqueo A, Gutiérrez J. Burden of care in families of patients with schizophrenia. Qual Life Res 2006; 15: 719-24. 
16. Urzúa A, Chirino A, Valladares G. Autoreporte de la Calidad de Vida Relacionada con la Salud en Diabetes Mellitus tipo 2. Rev Med Chile 2011; 139: 313-20.

17. Urzúa A, Pavlov N, Cortes R, Pino V. Factores psicosociales relacionados con la calidad de vida en pacientes hemodializados. Terapia Psicológica 2011; 29 (1): 135-40.

18. Fadda G, Jiron P. Calidad de vida y género en sectores populares Urbanos. Un estudio de caso en Santiago de Chile: Síntesis final y conclusiones. Boletín INVI 2001; 16: 105-38.

19. Browne F. Calidad de vida y entorno social, en dos comunas de zona central de Chile. Pharos 2004; 11 (2): 119-50.

20. Ming C. To weight or not to weight: the role of domain importance in quality of life measurement. Soc Indic Res 2004; 68: 163-74.

21. WHOQoL Group. Development of the World Health Organization WHOQOL-Bref Quality of Life Assessment. Psychological Medicine 1998; 28: 551-58.

22. Skevington S, Lotfy M, O'Conell K, The World Health Organization's WHOQOL-BREF quality of life assessment: Psychometric properties and results of the international field trial A report from the WHOQOL Group. Qual Life Res 2004; 13: 299-310.
23. Lucas-Carrasco R. The WHO quality of life (WHOQOL) questionnaire: Spanish development and validation studies. Qual Life Res 2012; 21: 161-65.

24. Mercier C, Péladeau N, Tempier R. Age, gender and quality of life. Community Mental Health Journal 1998; 34 (5): 487-500

25. Pucheu S, Consoli S, D`Auzac C, Francais P, Issad B. Do Health causal attributions and coping strategies act as moderators of quality of life in peritoneal dialysis patients. Journal of Psychosomatic Research 2004; 56: 317-22.

26. Urzúa A. Calidad de Vida y Factores Biopsicosociales en Patologías Médicas Crónicas. Terapia Psicológica 2008; 26 (2): 207-14.

27. Benyamini Y, Leventhal E, Leventhal H, Gender Differences in Processing Information for Making SelfAssessments of Health. Psychosomatic Medicine 2000; 62: 354-64.

28. Heinonen $\mathrm{H}$, Aro AR, Aalto AM, Uutela A. Is the evaluation of the global quality of life determined by emotional status. Qual Life Res 2004; 13: 1347-56.

29. Urzúa A, Jarne A. Calidad de vida y estilos de afrontamientos en personas con patologías crónicas. Revista Interamericana de Psicología 2008; 42 (1): 151-60. 